\title{
MANGÁ E A TRANSMISSÃO DE CULTURA: O EXEMPLO DE RUROUNI KENSHIN
}

Gustavo Furuyama

RESUMO: Este trabalho tem como objeto de estudo o mangá (história em quadrinhos japonesa) e sua utilização como instrumento de transmissão de cultura. Como base de análise foi utilizado o mangá "Rurouni Kenshin" Desta forma procurou-se identificar elementos que fazem referência à realidade e que podem ser usados como fonte de transmissão de conhecimento.

PALAVRAS -CHAVE: Mangá, Cultura Japonesa, Quadrinhos.

ABSTRACT: This work s subject is the Mangá (Japanese cartoon) and its use as a cultural transmission tool. The title chosen to be studied was "Rurouni Kenshin", that tells a history of a wandering samurai. In the analysis we tried to identify elements in the story that were related to the reality and could be used as a source of knowledge transmission.

KEYWORDS: Manga, Japanese culture, Cartoon.

Na sociedade moderna tudo se modifica e se moderniza com uma velocidade tão grande que às vezes nos sentimos sempre correndo atrás de algo. Como se existisse um princípio da obsolescência, em paródia ao princípio da incerteza, parece-nos que cada vez que compramos algo novo, o dinheiro injetado no sistema é usado para criar alguma coisa mais moderna.

Por esse ponto de vista tudo deveria evoluir e essa sua evolução seria o que decretaria a aposentadoria do seu antecessor. E isso realmente ocorre com carros, máquinas, computadores, etc. Felizmente, existem "coisas" que resistem ao tempo, entre elas as artes tradicionais. Quadros ainda são valiosos, a música clássica ainda é tocada com instrumentos medievais. Podemos arriscar dizer que, em geral, quando a idéia é mais importante do que o 
meio ou a mídia, esta tende a se manter resistente às mudanças, inversamente do que ocorre quando o meio é o produto final.

Não podemos tomar isso como uma certeza mas uma possibilidade que pode ajudar a explicar o objeto deste trabalho: o Mangá. Pelo tempo de existência dos mangás, em termos evolutivos pouco se fez no seu meio, mas talvez as idéias estejam mais contemporâneas. Um pequeno livro ou revista feito com papel jornal, monocromático, assim podemos definir o formato mais popular e tradicional do mangá, o tankohon(単行本). Porém, não podemos nos enganar, dentro dessa modesta aparência, podem-se esconder mundos e histórias que são, muitas vezes, mais complexas do que a vida real.

Em analogia à política romana do "pão e circo", no qual se dava ao povo comida (o pão) e diversão (o circo), o mangá seria como o circo para os japoneses. Às vezes até mais do que isso porque neles podemos encontrar fantasias, frustrações, história, cultura, sendo assim, não apenas um divertimento, mas um reflexo de uma sociedade. No Brasil ainda temos uma série de entraves para saber exatamente para que o mangá nos serve, sendo este um dos focos desta pesquisa.

Este trabalho tentou levantar dados e opiniões para mostrar o quanto o mangá pode ser visto como um meio de transmissão de conhecimento, entre eles a cultura ou até mesmo como instrumento educacional. Visto que existem milhares de títulos, foi escolhido um em particular, Rurouni Kenshin (Samurai X) para servir de exemplo e como objeto de teste para uma análise mais profunda do conteúdo que pode estar embutido junto com a trama principal.

Somente para ilustrar uma das funções iniciais do mangá, no Brasil, por um bom tempo os japoneses e seus descendentes o usavam para manter o idioma japonês atualizado. E, de fato, nos quadrinhos sempre se usam as últimas gírias, costumes e moda.

No Japão essa função de atualização da língua obviamente não existiu, então qual seria a função do mangá? Apenas diversão ou algo mais? Este trabalho tentará expor o ponto de vista dos japoneses em relação ao mangá, mostrando que algumas idéias são semelhantes às nossas, porém outras são um banho de água fria para nossas expectativas. Mesmo assim, essas diferenças são válidas para mostrar que muitas vezes estamos vivendo e relatando um mundo acadêmico que muitas vezes fica longe da realidade do povo. Não que algum japonês tenha dito "eu detesto mangá" mas a percepção de alguns ou a falta dela mostra que temos que tomar cuidado ao fazer análises de uma coisa que, de tão incrustada na cultura japonesa, pode ter usos e interpretações diferentes das que nós brasileiros podemos ver. Como o esquimó que enxerga uma dezena de tipos de branco, eles vêem o mangá por diversos ângulos, muitas vezes, ângulos que nunca enxergaremos. 
O mangá utilizado como referência se chama Rurouni Kenshin (conhecido como Samurai $\mathrm{X}$ no Brasil). Foram listados os eventos que se baseiam ou são fiéis à realidade do ponto de vista do autor. A reprodução fiel da história por si só pode não significar muita coisa para o mangá, e talvez o autor nem tenha se preocupado muito com isso, mas a interpretação que se tira dela ou da forma como os leitores percebem essa realidade é um dos focos deste estudo. Ao longo deste trabalho foram mostradas análises mais detalhadas de alguns aspectos como arquitetura, vestimenta, linguagem e técnica de desenho.

Com certeza, muitos fatos citados são muito conhecidos e podem existir diversos pontos de vista ou interpretações do mesmo, por isso, antes que surjam críticas, faremos algumas explicações. As informações citadas foram baseadas no que é contado na história existente no mangá Rurouni Kenshin. Por não se tratar de um livro sobre a história japonesa, e sim um mangá, os temas são tratados com caráter superficial e nem sempre fiéis aos livros oficiais.

Não cabe neste pequeno resumo a demonstração dos assuntos citados anteriormente, mas podemos deixar o exemplo da análise que foi feita, no tema recursos linguísticos. A língua japonesa se utiliza muito das onomatopéias e no mangá ela ganha muita visibilidade pela forma estilizada como ela é colocada. Existe até a contagem, que o próprio autor fez, de quantas vezes a palavra "oro oro" foi utilizada. Traduzido seria algo como "ai ai" no sentido de dor ou por ter feito alguma coisa errada, ou ainda, não saber o que falar ou fazer. Enfim, o "oro oro" foi dito 98 vezes ao longo da história e talvez seja uma das poucas onomatopéias que tenham sido traduzidas de forma literal.

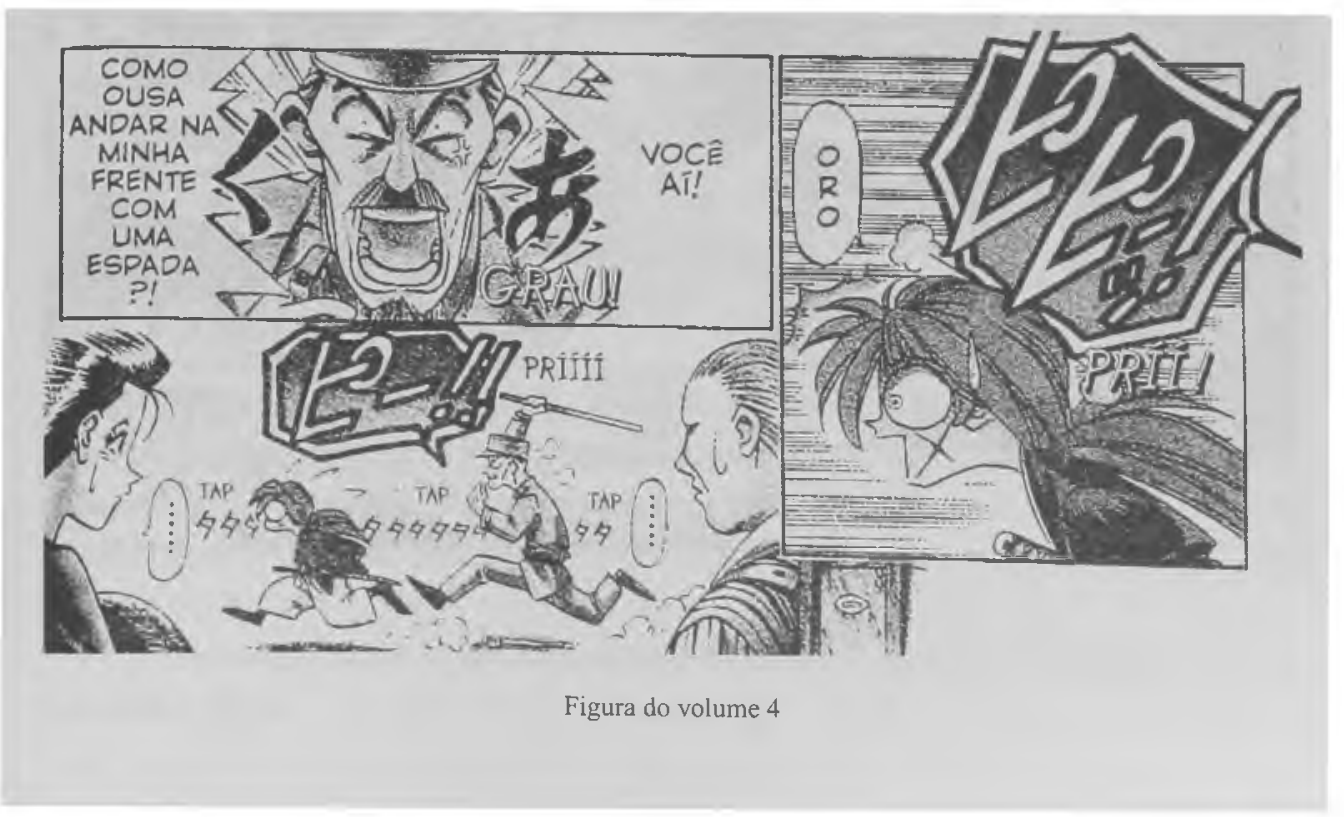


Talvez o que dê mais dinamismo ao mangá seja o uso visual da onomatopéia. Em japonês existem inúmeras onomatopéias, porém em português esse número é bem reduzido, o que talvez dificulte a tradução de algumas delas. Na versão inglesa nem existiu a tradução das onomatopéias e, em português, pode-se notar uma pequena transliteração junto ao texto original em japonês.

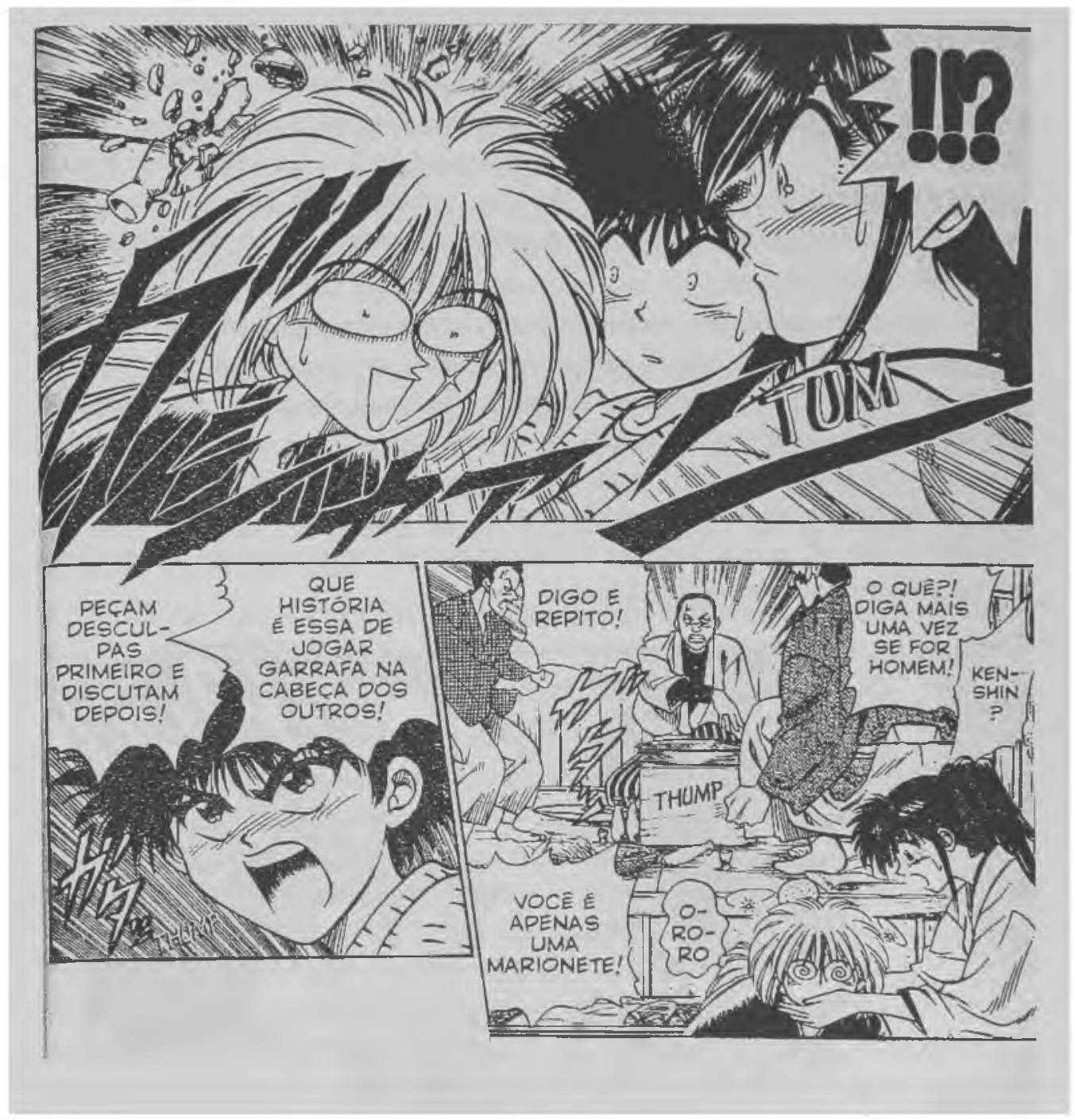

Figura do volume 1 
Na figura acima podemos ver que a palavra “ガジャアン”(gajyaan) foi adaptada para "Tum" Neste caso o uso foi relativamente correto, visto que teria que mostrar que houve um impacto. Porém, "gajyaan" pode ser entendido como um impacto que veio de algo que foi lançado, em movimento, enquanto que o "tum" nos parece algo um pouco seco, sem vida. A forma como o "gajyaan" está escrito também ajuda a entender o significado, a palavra tem "quinas" como alguma coisa que machuca e vem em forma de raio.

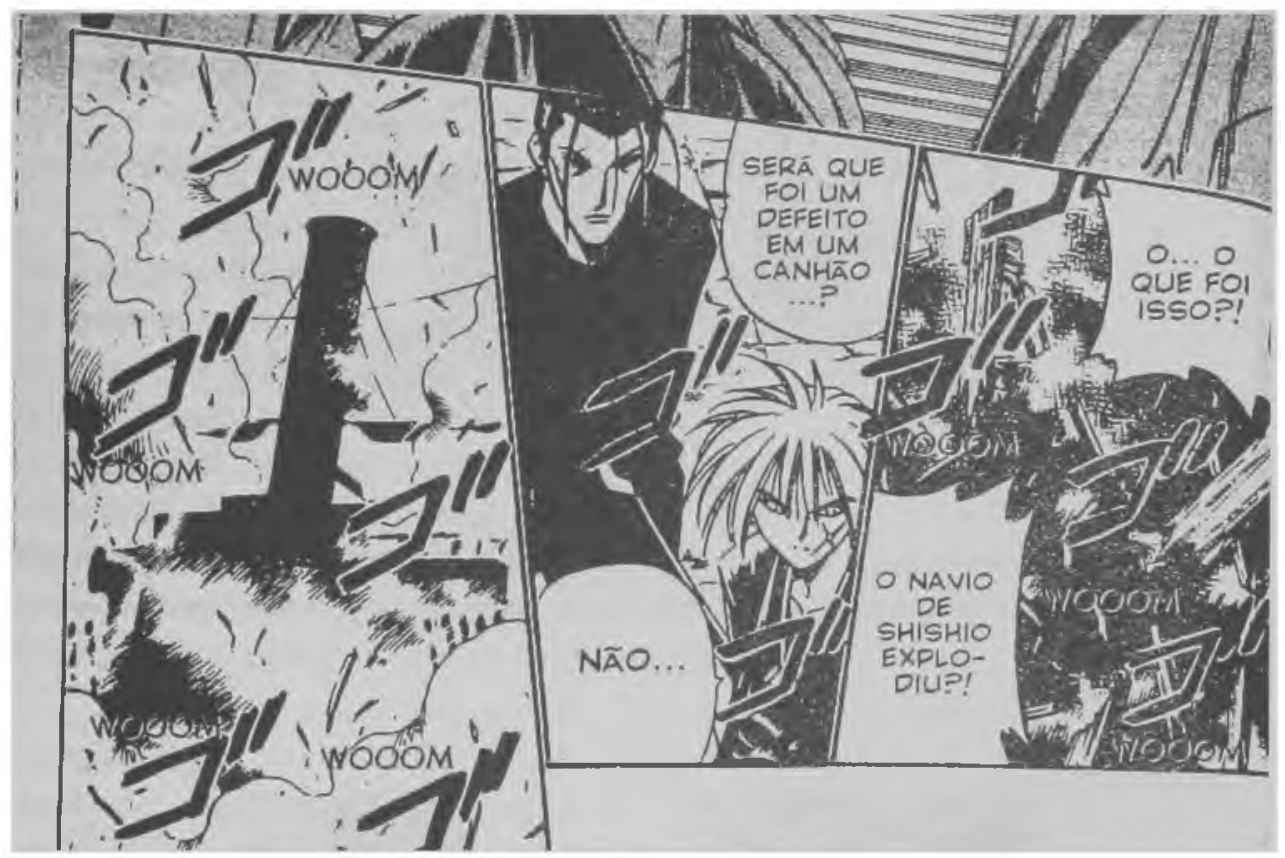

Figura do volume 12

Aqui temos outro exemplo, a onomatopéia “ت゙ゴゴ...” (go go go...) relativo ao motor ou barulho do navio foi adaptada como "wooom wooom..." Neste caso depende da interpretação pessoal para saber se chegamos perto do significado em japonês, mesmo porque "wooom" pode ser entendido como um barulho de carro, ou seja, longe de ser um estrondo de um navio.

Em outras traduções talvez o resultado tenha sido mais perto do esperado, como os exemplos a seguir. 


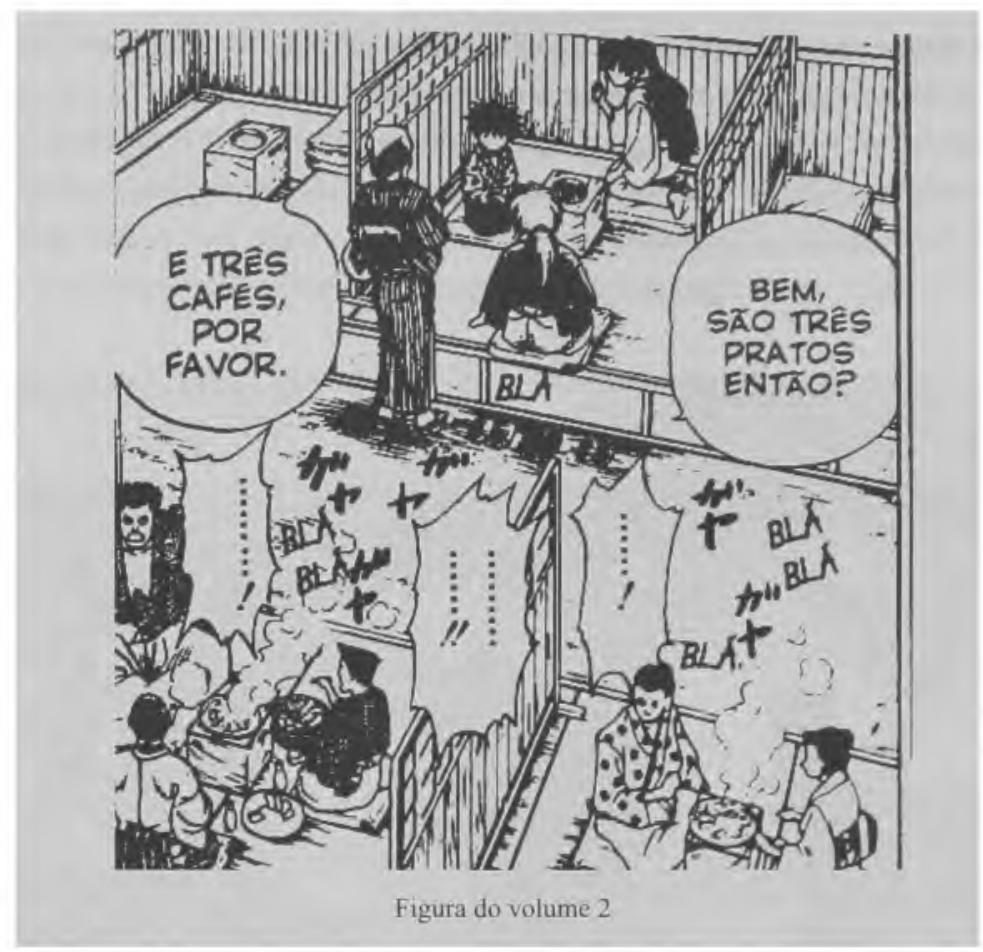

Neste exemplo temos o “ガヤ" (gaya) se repetindo, como se fosse um barulho de fundo, de pessoas conversando. A adaptação foi "blá blá..." o que corresponde bem ao original.

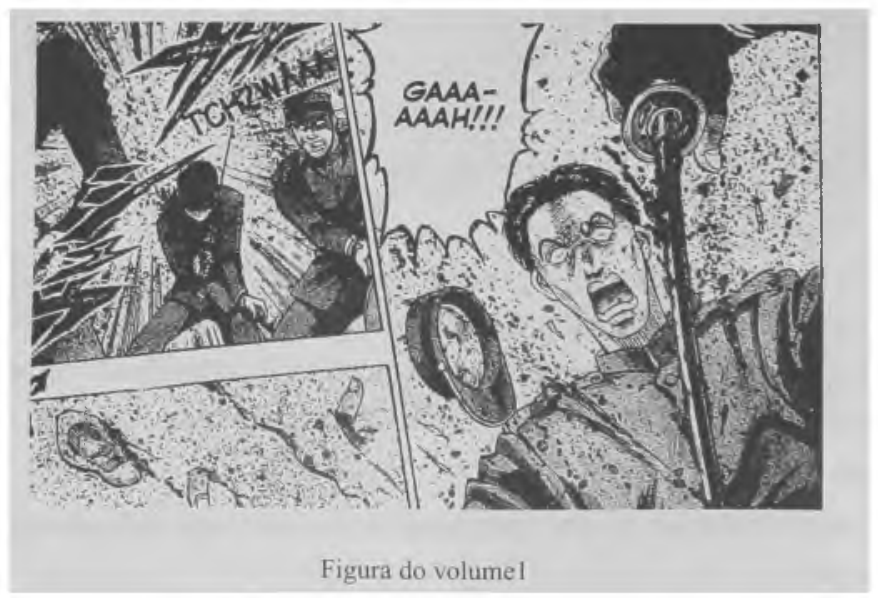


Em alguns casos temos adaptações inusitadas ou exóticas. Na figura anterior temos “ザ シュウ” (zasshuu) como se fosse uma lâmina cortando alguma coisa. O desenho com quinas da palavra nos remete a algo afiado, cortante. A tradução por sua vez foi "Tchzwaa" uma palavra que mal conseguimos pronunciar. Será mesmo esse o som de uma lâmina de espada?

Poderíamos ficar descrevendo cada onomatopéia que aparece no mangá mas isso criaria talvez outra dissertação, tamanha a sua extensão e complexidade. Por isso acreditamos que para este trabalho os exemplos citados sejam suficientes para explicar o que pode ocorrer na interpretação e adaptação dessas onomatopéias.

O universo do mangá é muito grande, portanto as conclusões deste trabalho refletem apenas uma pequena parte desse mundo dos quadrinhos que ainda é um campo muito pouco explorado. A intenção era mostrar uma das inúmeras maneiras que podemos entender $\mathrm{e}$ utilizar o mangá. Pudemos perceber semelhanças e diferenças em relação ao mangá no entendimento de uma pessoa japonesa e de uma pessoa não japonesa.

As semelhanças estão mais ligadas ao fato de que o mangá sempre está ligado ao entretenimento, à diversão. O estudo de mangá como uma mídia, como instrumento de ensino, ou como uma ferramenta de transmissão de cultura ainda é uma coisa muito incipiente no mundo inteiro, inclusive no Japão. Ainda é necessário vencer este pequeno preconceito para se poder trabalhar a parte teórica do mangá japonês. Talvez seja este o motivo que aproxima o entendimento do mangá no ocidente como um estilo, mais próximo às artes do que dos instrumentos de ensino. Não esperamos que o mangá tenha uma função semelhante ao do livro didático, mas seu conteúdo pode ser mais profundo do que mera diversão. Não sabemos com certeza ainda qual a profundidade à qual nos referimos, mas, com mais trabalhos na área, talvez possamos entender se estamos falando de uma poça ou de um poço.

Entender o mangá como um estilo foi uma mera simplificação ou resumo do que existe em termos de quadrinhos no Japão. Podem existir mangás com o traço idêntico aos dos quadrinhos da Marvel, e nem por isso deixariam de ser mangá. Entender o mangá como sendo apenas história em quadrinhos é uma característica japonesa. Já no ocidente entender como estilo é o mais comum, mas possivelmente, o que não se percebe a primeira vista, é que o conteúdo e a forma estrutural de uma mangá japonês é que fazem com que ele seja percebido como um produto tipicamente nipônico. Saberíamos que a história do battousai é japonesa, mesmo se eles estivessem desenhados como os X-men, por exemplo. E de fato muitos personagens da história analisada tinham "cara da Marvel"

No caso da história de Rurouni Kenshin podemos ver essa presença de conteúdo histórico associado a um traço identificado como japonês. Foi possível levantar inúmeros elementos que mostram a profundidade do embasamento para a criação da história de Kenshin. Desde a arquitetura à parte histórica, podemos ter boas referências da realidade que conhecemos. Para um leigo ou novato, as informações contidas no mangá podem ser utilizadas 
para conhecer e entender um pouco da cultura e história japonesa. Desta forma, diretamente ou não, existe uma transmissão de cultura, de conhecimento. Não consideramos a possibilidade de inserir títulos comerciais de mangá como leitura em sala de aula, mas imaginamos que seria possível exemplificar os textos dos livros didáticos com passagens existentes no mangá. A função de manutenção da língua japonesa para os imigrantes já não é muito usada pois a globalização permite que outros meios de ensino sejam mais eficazes que o mangá. Entretanto, o mangá ainda pode ser um modo de criar uma motivação para se estudar a língua japonesa. Um leitor que começa a ler os mangás japoneses deve perceber com o progresso da leitura que entender a língua japonesa é necessário caso ele queira entender a história por completo. Por isso podemos ver nas escolas de língua japonesa muitos não descendentes de japoneses querendo aprender a língua, em número igual ou muitas vezes superior do que os próprios nikkeis (descendente de japonês). Não sabemos o motivo da redução do número de nikkeis que estudam a língua japonesa, mas o mangá poderia ser utilizado para resgatar esse desejo de aprender a cultura e língua dos seus antepassados.

A língua é um elemento muito importante para se entender o mangá japonês. Infelizmente nem sempre é possível traduzir de forma coerente e completa todo o conteúdo presente no quadrinho. O desenho é uma parte muito importante do mangá, mas muitas vezes, como pudemos ver nas análises, a forma de como a língua é usada, principalmente com as onomatopéias, pode influenciar o modo como uma mensagem é transmitida. Para entendermos o superficial ou explícito bastariam os desenhos, mas os nuances e os detalhes da cultura japonesa talvez sejam apenas percebidos com uma compreensão completa do mangá, ou seja, da língua e da forma.

Este trabalho procurou analisar alguns aspectos que possibilitam iniciar uma análise conceitual e formal do mangá. Deste pequeno levantamento podemos concluir que o mangá é, sem dúvida, um instrumento muito completo que possibilita a transmissão de conhecimento. Cabe aos leitores identificar os pontos que podem ser úteis para determinada função. Possivelmente os japoneses não precisem, e talvez nem queiram, procurar uma função para os mangás, mas para os ocidentais tal classificação pode ser interessante. Por exemplo, se a função do mangá seria apenas diversão, basta uma compreensão superficial, ou seja, bastariam os desenhos e falas traduzidas de forma simples. Por outro lado, se a função é entender a cultura japonesa, seria necessária uma compreensão mais complexa do desenho, da língua, da estrutura do quadrinho, das implicações sociais e históricas.

Assim, seja para diversão ou para a difusão cultural, o mangá se estabelece como uma ferramenta muito versátil e de fácil penetração. Possivelmente o mangá possui qualidades e usos ainda não descritos e esperamos que, futuramente, mais trabalhos sejam feitos a seu respeito. Desta forma acreditamos que surgirão novas descobertas sobre essa excelente ferramenta que é o mangá. 
Bibliografia

AAKER, David A Brand leadership. Nova Iorque: The Free Press, 2000.

ARNOLD, Janet. Patterns of Fashion 2: Englishwomen's Dresses and Their

Construction C.1860-1940. Wace: Macmillan, 1972.

ASHELFORD, Jane. The Art of Dress: Clothing and Society 1500-1914. Abrams, 1996.

BAN, Toshio. Osamu Tezuka, uma biografia mangá. Conrad, 2004.

BARRAL, Étienne. Otaku os filhos do virtual. São Paulo: Editora SENAC, 2000.

ESCAJEDO, Javier. Las chicas son guerreras: Guía básica de Sailor Moon. Barcelona:

Camaleón Ediciones, 1998.

ESTUDIO FÉNIX. Cómo dibujar mangá. Martinez Roca. Barcelona, 1998.

Curso avanzado de mangá. Martínez Roca. Barcelona, 2001.

ESTUDIO INU. Dragon Ball: Dossier definitivo! Barcelona: Mafia Editores, 1995.

GALLEGO, Emilio. EI mundo dei mangá en Japón. Ediciones Veleta. Editora Granada, 2001.

GOLDTHORPE, Caroline. From Queen to Empress: Victorian Dress 1837-1877. Nova Iorque: Metropolitan Museum of Art, 1988.

GRAVETT, Paul. Mangá: como o Japão reinventou os quadrinhos. São Paulo: Conrad editora do Brasil, 2006.

GOTO Osamu. History of Japanese Architectures (日本建築史). Kyoritsu Shuppan (共 立出版), 2003.

HART, Christopher. MangáMania: HowtoDraw JapaneseComics. Nova Iorque: WatsonGuptill, 2001. HENSHIN n²1. Editora JBC. São Paulo, 2001.

INOUE, Manabu. Mangá no Yomikata (漫画の 読み方). Tóquio: Takarajima (宝島), 1995.

KINSELLA, Sharon. Adult Mangá: Culture \& Power in Contemporary Japanese Society. Londres: Curzon, 2000.

LAMBOURNE, Lionel. Japonisme: Cultural Crossings Between Japan and the West. Londres e Nova Iorque: Phaidon Press, 2005.

LEDOUX, Trish e RAMNEY, Doug. The Complete Anime Guide. Tiger Mountain Press. Washington: Issaquah, 1995 (nova edição em 1997).

LEVI, Antonia. Samurai from Outer Space: Understanding Japanese Animation. Chicago: Open Court Publishing, 1996.

LIDDELL, Jill. The History of the Kimono. Ep. Dutton, 1989.

LUYTEN, Sonia. Mangá, O Poder dos Quadrinhos Japoneses. São Paulo: Hedra, 2000 (primeira edição em 1991). 
McCARTHY, Helen. Anime! A Beginner's Guide to Japanese Animation. Londres: Titan Books, 1993.

.The Anime Movie Guide. Londres: Titan Books, 1996.

MIYAZAKI, Hayao. Master of Japanese Animation. Berkeley: Stone Bridge Press, 1999.

MCCLOUD, Scott. Desenhando Quadrinhos - os segredos das narrativas de quadrinhos, mangás e graphic novels. São Paulo: M. Books, 2008.

McCLOUD, Scott. Understanding Comics. Nova Iorque: Harper Collins Publishers Inc.1 (edição brasileira: Desvendando os Quadrinhos. Makron Books).

NAGADO, Alexandre. Almanaque da Cultura Pop Japonesa. São Paulo: Via Lettera, 2007

NAPIER, Susan J. Anime from Akira to Princess Mononoke. Nova Iorque: Palgrave, 2001.

NEWLAND, Amy Reigle. The Hotei Encyclopedia of Japanese Woodblock Prints. Amsterdã: Hotei Publishing, 2005.

NISHI, Kazuo; HOZUMI, Kazuo. What is Japanese Architecture.Tóquio: Kodansha International, 1985.

PEIRCE, Charles Sanders. Semiótica. Trad. J. Teixeira Coelho. São Paulo: Perspectiva, 1977.

PEKARIK, Julia Meech. The World of the Meiji Print. Nova Iorque: Weatherhill, 1986.

PEREZ, Clotilde. Signos da marca: expressividade e sensorialidade. São Paulo: Pioneira Thomson Learning, 2004.

RONI, Uever; YOSHIDA, Susugu. Ukiyo-E: 250 Years of Japanese Art. Gallery Books, 1991.

SANTAELLA, Lucia. Semiótica Aplicada. São Paulo: Pioneira Thomson Learning, 2004.

SANTAELlA, Lucia. A Teoria Geral dos Signos - Semiose e Autogeração. São Paulo: Ática, 1995.

SCHODT, Frederik L. Dreamland Japan. Berkeley: Stone Bridge Press, 1996.

SCHODT, Frederik L. Mangá! Mangá! The World of Japanese Comics. Tóquio: Kodansha International, 1983.

HORN, Maurice (coord.). The World Encyclopedia of Comics. Nova Iorque: ChE House, 1976 (nova edição atualizada em 1998).

WATSUKI, Nobuhiro. Kenshin Kaden. Tóquio: Shueisha Inc, 1999.

YABUNO, Ken; MIURA, Shumon. Meiji Kenchiku no Tabi (明治建築の旅). Tóquio: Shinchosha, 1988.

YAMADA, Chisaburoh F. Dialogue in Art: Japan and the West. Tóquio: Kodansha International Ltd, 1976. 